\title{
Erratum to: Visual analogue scales (VAS): Measuring instruments for the documentation of symptoms and therapy monitoring in cases of allergic rhinitis in everyday health care
}

\author{
Position Paper of the German Society of Allergology (AeDA) and the German \\ Society of Allergy and Clinical Immunology (DGAKI), ENT Section, in \\ collaboration with the working group on Clinical Immunology, Allergology and \\ Environmental Medicine of the German Society of Otorhinolaryngology, Head \\ and Neck Surgery (DGHNOKHC)
} Ludger Klimek $\cdot$ Karl-Christian Bergmann $\cdot$ Tilo Biedermann $\cdot$ Jean Bousquet $\cdot$ Peter Hellings $\cdot$ Kirsten Jung $\cdot$
Hans Merk $\cdot$ Heidi Olze $\cdot$ Wolfgang Schlenter $\cdot$ Philippe Stock $\cdot$ Johannes Ring $\cdot$ Martin Wagenmann $\cdot$
Wolfgang Wehrmann $\cdot$ Ralph Mösges $\cdot$ Oliver Pfaar

Published online: 27 January 2017

(c) Springer Medizin Verlag Berlin 2017

\section{Erratum to:}

Allergo J Int (2017)

DOI $10.1007 / \mathrm{s} 40629-016-0006-7$

In the online-first version of the article the following part of the main title was given as subtitle: "Measuring instruments for the documentation of symptoms and therapy monitoring in cases of allergic rhinitis in everyday health care”.
Additionally, the subtitle was mistakenly set next to the author affiliations: "Position Paper of the German Society of Allergology (AeDA) and the German Society of Allergy and Clinical Immunology (DGAKI), ENT Section, in collaboration with the working group on Clinical Immunology, Allergology and Environmental Medicine of the German Society of Otorhinolaryngology, Head and Neck Surgery (DGHNOKHC)". The original article was corrected.
The online version of the original article can be found under http://dx.doi.org/10.1007/s40629-016-0006-7.

Prof. Dr. med. L. Klimek $(\bowtie) \cdot$ O. Pfaar

Center for Rhinology and Allergology, An den

Quellen 10, 65183 Wiesbaden, Germany

Ludger.Klimek@Allergiezentrum.org

\section{K.-C. Bergmann}

Charité Allergy Center, Department of Dermatology,

Venereology, and Allergology, Charité, Berlin, Germany

T. Biedermann $\cdot$ J. Ring

Department and Outpatient Clinic for Dermatology and

Allergology am Biederstein, Technical University of Munich,

Munich, Germany
J. Bousquet
CHRU, Montpellier University Hospital Center, Montpellier, France

MACVIA-LR, Montpellier, France

INSERM, VIMA, Université Versailles

St.-Quentin-en-Yvelines, Versailles, France

P. Hellings

Institute for Otorhinolaryngology, Leuven University Hospital, Leuven, Belgium

Institute für Otorhinolaryngology, Academic Medical Center, Amsterdam, The Netherlands 


\section{K. Jung}

Group Practice for Dermatology, Erfurt, Germany

H. Merk

Department of Dermatology and Allergology - Department of Dermatology, RWTH University Hospital, Aachen,

Germany

H. Olze

ENT Department, Charité University Hospital, Berlin, Germany

\section{W. Schlenter}

Formerly: Otorhinolaryngology, St. Marien Hospital,

Frankfurt, Germany

P. Stock

Pediatric Pneumology and Allergology, AKK Altonaer

Children's Hospital, Hamburg, Germany

J. Ring

Christine Kühne Center for Allergy Research and Education (CK-Care), Davos, Switzerland

M. Wagenmann

Ear, Nose, and Throat Department, Düsseldorf University Hospital, Düsseldorf, Germany

W. Wehrmann

Dermatology Group Practice, Münster, Germany

R. Mösges

Institute of Medical Statistics, Informatics and

Epidemiology, Medical Faculty, University of Cologne,

Cologne, Germany

O. Pfaar

Department of Otorhinolaryngology, Head and Neck

Surgery, Medical Faculty Mannheim, Universitätsmedizin

Mannheim, Heidelberg University, Mannheim, Germany 